\title{
Dilatonic Equation of Hydrostatic Equilibrium and Neutron Star Structure
}

\author{
S. H. Hendi ${ }^{1,2}$ - G. H. Bordbar ${ }^{1,2}$ • \\ B. Eslam Panah ${ }^{1}$ • M. Najafi ${ }^{1}$
}

\begin{abstract}
In this paper, we present a new hydrostatic equilibrium equation related to dilaton gravity. We consider a spherical symmetric metric to obtain the hydrostatic equilibrium equation of stars in 4-dimensions, and generalize TOV equation to the case of regarding a dilaton field. Then, we calculate the structure properties of neutron star using our obtained hydrostatic equilibrium equation employing the modern equations of state of neutron star matter derived from microscopic calculations. We show that the maximum mass of neutron star depends on the parameters of dilaton field and cosmological constant. In other words, by setting the parameters of new hydrostatic equilibrium equation, we calculate the maximum mass of neutron star.
\end{abstract}

Keywords hydrostatic equilibrium of stars; dilaton gravity; neutron star; maximum mass

\section{Introduction}

Our observations of Supernovae type Ia (Riess et al. 2004; Perlmutter et al. 1999a; Perlmutter et al. 1999b) confirm that the expansion of our Universe is currently undergoing a period of acceleration. But Einstein (EN) gravity can not explain this acceleration. In addition, although Einstein's theory can explain the solar system phenomena successfully, when we want to study beyond the solar system or when the gravity is so strong,

\footnotetext{
S. H. Hendi

G. H. Bordbar

B. Eslam Panah

M. Najafi

${ }^{1}$ Physics Department and Biruni Observatory, College of Sciences, Shiraz University, Shiraz 71454, Iran

${ }^{2}$ Research Institute for Astronomy and Astrophysics of Maragha (RIAAM), Maragha, Iran
}

this theory encounters with some problems, and so we need to modify EN gravity. In order to improve EN gravity, one may add a (cosmological) constant to its Lagrangian (Padmanabhan 2003; Frieman 2008). Moreover, we can regard other modifications of Einstein gravity such as Lovelock gravity (Lovelock 1971; Lovelock 1972; Deruelle 1990; Hendi 2008), brane world cosmology (Demetrian 2006; Brax 2003; Gergely 2006; Amarilla 2010), scalar-tensor theories (Jordan 1955; Brans 1961; Cai 2007; Fujii 2003; Sotiriou 2006; Giddings 1993; Gregory 1997; Ling 2013; Klepac 2002; Ghodrati 2008) and $F(R)$ gravity (Bamba 2008; Cognola 2008; Corda 2009; Sotiriou 2010; Nojiri 2011; Hendi 2012; Hendi 2014a; Momeni 2014).

On the other hand, dark energy and dark matter have received a lot of attention in recent years. Theoretical physicists introduced non-baryonic (Dick 1996) dark matters with three models of cold, warm and hot. Among them cold dark matter model has the highest agreement with the experimental observations. It is worthwhile to mention that, dilaton field is one of the most interesting candidates for cold dark matter (Cho 1990). In addition, one of the the best approaches for finding the nature of dark energy is taking into account a new scalar field (Huang 2007; Huang 2008). Moreover, the low energy limit of string theory contains a dilaton field which is coupled to gravity. Physical properties, thermodynamics, and thermal stability of the black object solutions in the context of dilaton theory have been investigated before (Tamaki 2000; Yamazaki 2001; Yazadiiev 2005; Dehghani 2008).

The hydrostatic equilibrium equation (HEE) plays crucial role in studying the evolution of the stars. This equation is giving an insight regarding the equilibrium state between internal pressure and gravitational force of the stars.

It is important to note that the neutron and quark stars have large amount of mass concentrated in small 
radius. Therefore, they are in the category of highly dense objects, the so-called compact stars. Due to this fact, we need to take into account the effects of general relativity such as the curvature of spacetime for studying the compact stars. The first HEE in 4-dimensional Einstein gravity was studied by Tolman, Oppenheimer and Volkoff (TOV) (Tolman 1934; Tolman 1939; Oppenheimer 1939). Also, the physical characteristics of stars using TOV equation have been investigated in Refs. (Silbar 2004; Narain 2006; Bordbar 2006a; Bordbar 2006b; Li 2012; Bordbar 2013; Yazdizadeh 2013; Oliveira 2014). On the other hand, if one is interested in studying the structure and evolution of stars in different gravities, one should obtain the HEE in those gravity models. In recent years, the generalizations and modifications of this equation were of special interests for many authors (Hevdarifard 2009; Astashenok 2013; Orellana 2013; Arbanil 2013; Doneva 2013; Goswami 2014; Lemos 2015; Yazadiiev 2014; Capozziello 2011; Capozziello 2012; Momeni 2015a). For more examples, we may note that the HEE equation in $f(R)$ and $f(G)$ gravities werediscussed in Astashenok 2015b; Momeni 2015b; Astashenok 2015a; Abbas 2015), $d$-dimensional HEE in EN gravity was investigated in (Ponce 2000) and HEE of EN- $\Lambda$ gravity with arbitrary dimensions was obtained in (Bordbar 2015). In addition, 5 and higher dimensional HEE in context of Gauss-Bonnet (GB) gravity was extracted in (Zhan-Ying 2012; Hansraj 2015; Bordbar 2015). Recently, (2+1)-dimensional HEE was obtained for a static star in the presence of cosmological constant (Diaz 2014).

In this paper, we want to obtain modified HEE in the presence of dilaton field. We consider the Lagrangian of Einstein-dilaton gravity and a perfect fluid energymomentum tensor with spherical symmetric metric to obtain dilatonic HEE. We also consider dilaton field as a correction of Einstein gravity to obtain a perturbative HEE. In other words, we obtain HEE for Einstein gravity with an additional term which the trace of small dilaton field.

\section{Equation of hydrostatic equilibrium with a dilaton field}

The action of dilaton gravity in the context of Einstein gravity is given by

$I_{G}=\frac{1}{16 \pi} \int d^{4} x \sqrt{-g}\left[R-2 g^{\mu \nu} \partial_{\mu} \Phi \partial_{\nu} \Phi-V(\Phi)\right]+I_{M}$,

where $R$ and $\Phi$ are, respectively, the Ricci scalar and the dilaton field. Also $V(\Phi)$ is the potential for $\Phi$, and
$I_{M}$ denotes the action of matter field which we consider a perfect fluid. Varying the action (10) with respect to the metric tensor $g_{\mu}^{\nu}$ and the dilaton field $\Phi$, the equations of motion for this gravity can be written as

$$
\begin{aligned}
R_{\mu}^{\nu}-\frac{1}{2} g_{\mu}^{\nu} R= & 2 \partial_{\mu} \Phi \partial^{\nu} \Phi-\frac{1}{2} g_{\mu}^{\nu} V(\Phi) \\
& -g_{\mu}^{\nu} \partial_{c} \Phi \partial^{c} \Phi+K T_{\mu}^{\nu}, \\
\nabla^{2} \Phi= & \frac{1}{4} \frac{\partial V}{\partial \Phi}
\end{aligned}
$$

where $K=\frac{8 \pi G}{c^{4}}$. In order to construct consistent solutions of the field equations (2) and (3), we adopt the approach of gravitational papers (Chan 1995) and assume that the dilaton potential contains two Liouville terms

$V(\Phi)=2 \Lambda_{0} e^{2 \xi_{0} \Phi}-2 \Lambda e^{2 \xi \Phi}$,

where $\Lambda_{0}, \Lambda, \xi_{0}$, and $\xi$ are constants. This kind of potential was previously investigated in (Chan 1995).

In the present work, we want to obtain the static solutions of Eq. (2). So, we assume the spacetime metric has the following form

$d s^{2}=f(r) d t^{2}-\frac{d r^{2}}{g(r)}-r^{2} R^{2}(r)\left(d \theta^{2}+\sin ^{2} \theta d \varphi^{2}\right)$,

where $f(r), g(r)$ and $R(r)$ are functions of $r$ which should be determined.

The equations (2) and (3) contain four unknown functions $f(r), g(r), R(r)$ and $\Phi(r)$. In order to solve them, we consider the ansatz

$R(r)=e^{\alpha \Phi(r)}$.

This ansatz was first introduced in (Dehghani 2005) for the purpose of finding black string solutions of Einstein Maxwell dilaton gravity. It is notable that in the absence of the dilaton field ( $\alpha=0$ and so $R(r)=1$ ), dilaton gravity reduces to EN gravity. Also, using Eqs. (3) and (6) and the metric introduced in Eq. (5), we can obtain

$\Phi(r)=\frac{\alpha}{\mathcal{K}_{1,1}} \ln \left(\frac{b}{r}\right)$,

where $b$ is an arbitrary constant and $\mathcal{K}_{i, j}=i+j \alpha^{2}$.

On the other hand, the energy-momentum tensor for a perfect fluid is

$T^{\mu \nu}=\left(P+\rho c^{2}\right) U^{\mu} U^{\nu}+P g^{\mu \nu}$,

where $P$ and $\rho$ are, respectively, pressure and density of the fluid which are measured by the local observer, and $U_{\mu}$ is the fluid four-velocity. Using Eq. (8) and 
the metric introduced in Eq. (5), we can obtain the components of energy-momentum for $(3+1)$-dimensions as follows

$T_{0}^{0}=\rho c^{2} \quad \& \quad T_{1}^{1}=T_{2}^{2}=T_{3}^{3}=-P$.

Now, we consider the metric (5) and Eq. (9) for the perfect fluid to obtain the components of Eq. (2) with the following forms

$$
\begin{aligned}
K \rho c^{2}= & \frac{\left(1-r^{2} \Lambda \Upsilon^{2 \alpha^{2}}\right)}{\Upsilon^{\alpha^{2}} r^{2}}+\frac{\alpha^{2} \Upsilon}{b^{2} \mathcal{K}_{-1,1}} \\
& -\frac{\left(g+r g^{\prime} \mathcal{K}_{1,1}\right)}{r^{2} \mathcal{K}_{1,1}^{2}}, \\
K P= & -\frac{\left(1-r^{2} \Lambda \Upsilon^{2 \alpha^{2}}\right)}{\Upsilon^{\alpha^{2}} r^{2}}-\frac{\alpha^{2} \Upsilon}{b^{2} \mathcal{K}_{-1,1}} \\
& +\frac{g\left(f+r f^{\prime} \mathcal{K}_{1,1}\right)}{r^{2} f \mathcal{K}_{1,1}^{2}}, \\
K P= & -\frac{\left(4 f \Lambda \Upsilon^{\alpha^{2}}-2 g f^{\prime \prime}-f f^{\prime}\left(\frac{g}{f}\right)^{\prime}\right)}{4 f} \\
& -\frac{\alpha^{2} \Upsilon}{b^{2} \mathcal{K}_{-1,1}}+\frac{(f g)^{\prime}}{2 r f \mathcal{K}_{1,1}}+\frac{\alpha^{2} g}{r^{2} \mathcal{K}_{1,1}^{2}},
\end{aligned}
$$

where

$\Upsilon=\left(\frac{b}{r}\right)^{\frac{2}{\kappa_{1,1}}}$,

and $f, g, \rho$ and $P$ are functions of $r$. It is notable that the prime and double prime are, respectively, the first and second derivatives with respect to $r$. On the other hand, substituting $\alpha=0$ in Eqs. (10][12), one finds the corresponding field equations of EN gravity (see (Bordbar 2015) for more details).

Using Eqs. (10 12) and after some algebraic calculations, we obtain

$$
\begin{aligned}
& \frac{d P}{d r}+\frac{f^{\prime}\left(c^{2} \rho+P\right) \mathcal{K}_{1,-1}}{2 f}+\frac{2 \alpha^{2}\left(1-r^{2} \Lambda \Upsilon^{2 \alpha^{2}}\right)}{K \Upsilon^{\alpha^{2}} r^{3} \mathcal{K}_{1,1}}- \\
& \frac{2 \alpha^{2} \Upsilon}{r K b^{2} \mathcal{K}_{1,1} \mathcal{K}_{-1,1}}+\frac{\alpha^{2} g f^{\prime \prime}}{r f \mathcal{K}_{1,1}}+\frac{\alpha^{2}\left(r g^{\prime}+2 g\right)}{r^{3} \mathcal{K}_{1,1}^{2}}=0 .(14)
\end{aligned}
$$

In addition, one can use Eq. (11) to obtain $f^{\prime}$ with the following form

$$
\begin{aligned}
& f^{\prime}= \frac{r f\left(\frac{\Upsilon^{-\alpha^{2}}}{r^{2} g}+\Lambda \Upsilon^{\alpha^{2}}+K P\right) \mathcal{K}_{1,1}}{g} \\
&+\frac{r \alpha^{2} \Upsilon \mathcal{K}_{1,1} f}{g b^{2} \mathcal{K}_{-1,1}}-\frac{f}{r \mathcal{K}_{1,1}}
\end{aligned}
$$

To obtain the function $g(r)$, we consider Eq. (10). After integration we achieve

$$
\begin{aligned}
g(r)= & \left(\frac{\alpha^{2} \Upsilon}{b^{2} \mathcal{K}_{1,2} \mathcal{K}_{1,-1}}+\frac{\Lambda \Upsilon^{\alpha^{2}}}{3}+\frac{1}{\Upsilon^{\alpha^{2}} r^{2} \mathcal{K}_{1,2}}\right) \mathcal{K}_{1,1}^{2} r^{2} \\
& -\frac{K c^{2} \mathcal{K}_{1,1}}{r^{\frac{1}{\kappa_{1,1}}}} \int \rho(r, \alpha) r^{\frac{\mathcal{K}_{2,1}}{\mathcal{K}_{1,1}}} d r
\end{aligned}
$$

where $\rho(r, \alpha)=\frac{d M(r, \alpha)}{d V_{e f f}}$, in which $V_{\text {eff }}=\frac{4}{3} \pi R_{\text {eff }}^{3}$ and $R_{\text {eff }}=\left(\frac{3 \mathcal{K}_{1,1}}{\mathcal{K}_{2,3}}\right)^{1 / 3}{ }^{1 / \mathcal{K}_{2,3} / 3 \mathcal{K}_{1,1}}$. It is interesting to note that in the presence of dilaton field we should replace $r$ with $R_{\text {eff }}$. In other words, dilaton field can modify the radius of sphere into $R_{\text {eff }}$ instead of usual sphere with radius $r$. It is notable that, when $\alpha=0$ (in the absence of the dilaton field), $R_{\text {eff }}$ reduces to $r$ and also (as we expect) we obtain $g(r)=1-\frac{\Lambda}{3} r^{2}-\frac{m(r)}{4 \pi r}$, where $m(r)=\int 4 \pi r^{2} \rho(r) d r$.

Now, we consider the integral that appears in Eq. (16) and by using of $R_{e f f}$, the equation (16) turns into

$$
\begin{aligned}
g(r)= & \left(\frac{\alpha^{2} \Upsilon}{b^{2} \mathcal{K}_{1,2} \mathcal{K}_{1,-1}}+\frac{\Lambda \Upsilon^{\alpha^{2}}}{3}+\frac{1}{\Upsilon^{\alpha^{2}} r^{2} \mathcal{K}_{1,2}}\right) \mathcal{K}_{1,1}^{2} r^{2} \\
& -\frac{K c^{2} \mathcal{K}_{1,1}}{4 \pi r^{\frac{1}{\mathcal{K}_{1,1}}}} M_{\text {eff }}(r, \alpha),
\end{aligned}
$$

where we used $M_{\text {eff }}(r, \alpha)=\int \rho(r, \alpha) 4 \pi R_{\text {eff }}^{2} d R_{e f f}$. It is notable that, $M_{\text {eff }}(r, \alpha)$ and $R_{\text {eff }}$ are, respectively, the effective mass and radius as results of the presence of the dilaton field. In obtained solution (17) and for consistency we use

$\xi_{0}=\frac{1}{\alpha}, \quad \xi=\alpha, \quad \Lambda_{0}=\frac{\alpha^{2}}{b^{2} \mathcal{K}_{-1,1}}$.

Notice that $\Lambda$ remains as a free parameter which plays the role of the cosmological constant.

Now, we can obtain the HEE for dilaton gravity. For this purpose, we consider the Eqs. (15) and (17), and inserting them in Eq. (14). After some manipulation we obtain

$$
\frac{d P}{d r}=\frac{\alpha^{2}\left(\mathcal{A} c^{2}-\mathcal{B} \mathcal{K}_{1,1}^{2}+\mathcal{C}+\mathcal{D}\right)}{K r^{3} f \mathcal{K}_{1 / 2,1} \mathcal{K}_{-1,1} \mathcal{K}_{1,1}^{3}}
$$

where $\mathcal{A}, \mathcal{B}, \mathcal{C}$ and $\mathcal{D}$ are in the following forms

$$
\begin{aligned}
\mathcal{A} & =\frac{K}{4 \pi r^{1 / \mathcal{K}_{1,1}}} \mathcal{K}_{1,1} \mathcal{K}_{-1,1} \mathcal{K}_{1 / 2,1} \mathcal{X}_{1,2,1} M_{\text {eff }}(r, \alpha), \\
\mathcal{B} & =\frac{r^{2}}{3}\left(\Lambda \mathcal{K}_{-1,1} \mathcal{K}_{1 / 2,1} \gamma^{\alpha^{2}} \mathcal{X}_{2,1,2}+\frac{3 \alpha^{2} \gamma \mathcal{X}_{1,1,2}}{2 b^{2}}\right), \\
\mathcal{C} & =2 \gamma^{\alpha^{2}} \mathcal{K}_{1,1}^{2} \mathcal{K}_{-1,1}\left(\Lambda r^{2} f \mathcal{K}_{1 / 2,1}-\frac{\mathcal{X}_{1,2,4}}{4 \gamma^{2 \alpha^{2}}}\right), \\
\mathcal{D} & =r^{2} f \mathcal{K}_{1,1}^{2} \mathcal{K}_{1 / 2,1}\left[K c^{2} \rho \mathcal{K}_{-1,1}^{2}+\left(P+\rho c^{2}\right) \mathcal{Y}\right],
\end{aligned}
$$


and also $\mathcal{X}_{i, j, k}=r^{2} \mathcal{K}_{1,1}^{2} f^{\prime \prime}+k \mathcal{K}_{i, j} f \quad$ and $\mathcal{Y}=\frac{2 \gamma}{b^{2}}+$ $\frac{K r f^{\prime}}{2 \alpha^{2} f} \mathcal{K}_{1,1}^{2} \mathcal{K}_{-1,1}^{2}$.

In next section, we continue our paper with considering dilaton gravity as a correction (perturbation) of Einstein gravity and we will obtain the corresponding HEE.

\section{Dilaton gravity as a correction of Einstein gravity}

The interesting agreements and acceptable results of the EN gravity with experimental results (observations) guide us to consider its modification (such as dilaton gravity) as a correction of EN gravity. On the other hand, to avoid the complexity of modified gravity theories and obtaining credible solutions, it is logical to consider the dominant perturbative terms. Therefore in this section, we discuss the effects of small value $\alpha$. When $\alpha$ is very small, we can use series expansion in Eq. (18) and keep the dominant contribution term. So, we keep $\mathcal{O}\left(\alpha^{2}\right)$ and ignore $\mathcal{O}\left(\alpha^{4}\right)$ and higher orders to obtain

$$
\begin{aligned}
\frac{d P}{d r}= & \frac{\left[3 K c^{2} m(r)+4 \pi r^{3}(3 K P+2 \Lambda)\right]}{r^{2}\left[3 K c^{2} m(r)-4 \pi r\left(\Lambda r^{2}+3\right)\right]}\left(P+\rho c^{2}\right)+ \\
& \frac{3\left(P+\rho c^{2}\right) \mathcal{H} \alpha^{2}}{\mathcal{K}_{1,1}\left[r\left(\Lambda r^{2}+3\right)-\frac{3 K c^{2} m(r)}{4 \pi}\right]^{2}}+\mathcal{O}\left(\alpha^{4}\right), \quad(19)
\end{aligned}
$$

where $\mathcal{H}$ is

$$
\begin{aligned}
\mathcal{H}= & \frac{3 K c^{2}\left[1+r^{2}(\Lambda+K P)\right][4 \pi \mathcal{M}(r)-m(r)]}{8 \pi} \\
& +\frac{9 K^{2} c^{6} m^{3}(r)}{64 \pi^{3} r^{4}\left(P+\rho c^{2}\right)}-\frac{3 K c^{2} m(r) \varpi_{1}}{4 \pi} \\
& -\frac{3 K c^{4} m^{2}(r)\left[12+r^{2}(2 \Lambda+K P)\right]}{16 \pi^{2} r^{3}\left(P+\rho c^{2}\right)}+\varpi_{2},
\end{aligned}
$$

in which

$$
\begin{aligned}
\varpi_{1}= & \frac{P\left[\ln r-5-\Lambda r^{2}\left(\frac{10}{3}-\ln r\right)\right]}{2\left(P+\rho c^{2}\right)} \\
& +\frac{c^{2} \rho\left[\left(\Lambda r^{2}+1\right) \ln r-2 \Lambda r^{2}-1\right]}{2\left(P+\rho c^{2}\right)} \\
& -\left(r^{2} \Lambda-1\right) \ln \left(\frac{b}{r}\right)+K r^{2} P\left(\frac{\ln r}{2}-1\right) \\
& -\frac{18 \Lambda r^{2}+\Lambda^{2} r^{4}+45}{3 K r^{2}\left(P+\rho c^{2}\right)} \\
\varpi_{2}= & r^{3}\left(c^{2} \rho+P\right)\left[K P\left(\Lambda r^{2}-3\right)-4 \Lambda\right] \ln \left(\frac{b}{r}\right) \\
& -\frac{P r\left[\frac{2 \Lambda^{2} r^{4}}{3}+9 K c^{2} r^{2} \rho+12\left(1+\Lambda r^{2}\right)\right]}{2} \\
& -\frac{2\left(\Lambda r^{2}+3\right)^{2}}{K r}-\frac{9 K r^{3} P^{2}}{2} \\
\mathcal{M}(r)= & -c^{2} r \rho\left(4 \Lambda r^{2}+3\right), \\
& \int r^{2} \ln (r) \rho(r) d r .
\end{aligned}
$$

It is notable that, as one expects the first term of Eq. (19) is the TOV equation in the presence of cosmological constant. In addition, the second term $(\mathcal{H})$ is the leading order term of considered dilaton field as a correction to EN gravity.

We are going to continue our paper with considering HEE obtained in Eq. (19) and obtain the properties of neuron stars by using of cluster expansion of the energy.

\section{Structure properties of neutron star}

\subsection{Equation of state of neutron star matter}

In order to study properties of the neutron star structure, we need to consider an equation of state for neutron star matter. The constituents of interior part of a neutron star are neutrons, protons, electrons and muons which are in charge neutrality and beta equilibrium conditions (beta-stable matter) (Shapiro 1983). In recent years, we have obtained the equation of state of neutron star matter using the microscopic constrained variational calculations based on the cluster expansion of the energy functional Bordbar 2002; Bordbar 2004; Bordbar 2006a). In these studies the modern two-nucleon potentials such as the new Argonne $A V_{18}$ (Wiringa 1995) and charged dependent Reid-93 (Stoks 1994) have been used. One of the importances of these calculations is the fact that it does not bring any free parameter into the formalism, and 


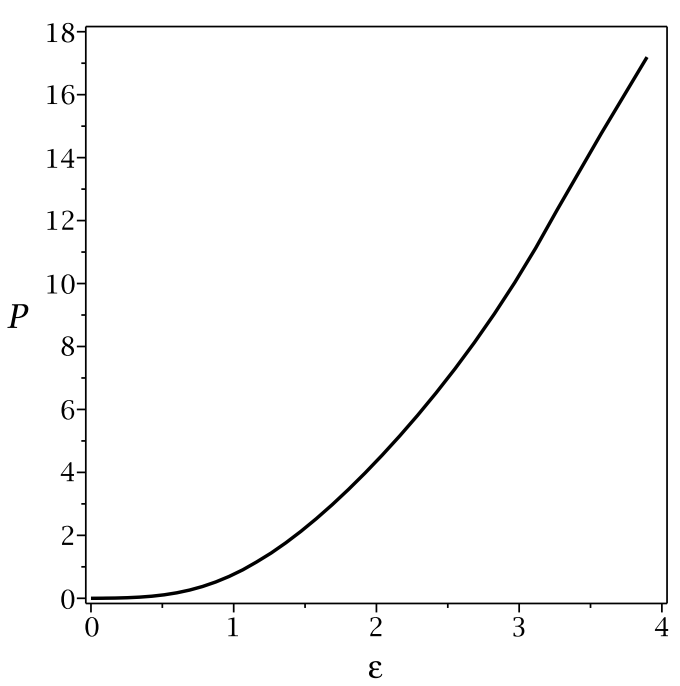

Fig. 1 Equation of state of neutron star matter (pressure, $P\left(10^{35} \mathrm{erg} / \mathrm{cm}^{3}\right)$ versus density, $\left.\epsilon\left(10^{15} \mathrm{gr} / \mathrm{cm}^{3}\right)\right)$.

its results show a good convergence. In this method, a microscopic computation of asymmetry energy is carried on for the asymmetric nuclear matter calculations which results into more accuracy in comparison with other semi-empirical parabolic approximation methods. In fact, a microscopic calculation is required with the modern nucleon-nucleon potentials which are explicitly depend on the isospin projection $\left(T_{z}\right)$ (Bordbar 1998).

In this paper, for neutron star structure, we use the modern equation of state which has been calculated using $A V_{18}$ potential (Bordbar 2002; Bordbar 2004; Bordbar 2006a) and investigate some physical properties of neutron star structure. This equation of state of neutron star matter is shown in Fig. 1.

\subsection{Maximum mass of neutron star}

In order to distinguish neutron stars from black holes, it is important to know the maximum gravitational mass of a neutron star for stability against collapse into a black hole. In other words, it is expected that below a certain maximum mass, degenerate pressure due to the nucleons is sufficient to prevent an object from becoming a black hole (Shapiro 1983). Therefore, determining the maximum gravitational mass of neutron stars is of special importance in astrophysics. Direct ways to measure the neutron star mass are observations of the X-ray pulsars and X-ray bursters, but there are many errors in these methods and so, measuring the mass of neutron star is not very useful and accurate. Fortunately, the mass of neutron stars have been determined with high accuracy using the binary radio pulsars (Weisberg 1984; Liang 1986; Heap 1992; Jonker 2003; Quaintrell 2003).
Here, we calculate the maximum mass of neutron star using the equation of state of neutron star matter presented in Fig. 1 by numerical integrating the HEE obtained in Eq. (19). This leads to the neutron star mass and radius as a function of central mass density $\left(\epsilon_{c}\right)$. For this purpose, by selecting a central mass density $\left(\epsilon_{c}\right)$, under the boundary conditions $P(r=0)=P_{c}$ and $m(r=0)=0$, we integrate the Eq. (19) outwards to a radius $r=R$ in which $P$ vanishes. This yields the neutron star radius $R$ and mass $M=m(R)$. Our results have been given in the following figures and tables.

Our results show that the maximum mass and radius of neutron stars depend on the parameters of dilaton field $(\alpha)$ and cosmological constant $(\Lambda)$. As one can see in Table 1, we have obtained the maximum mass and radius of neutron star in the absence of cosmological constant. The results show that as $\alpha$ increases, the maximum mass of neutron star decreases. We have found that the maximum mass of neutron star is affected with variation of $\alpha$ for the case of $\alpha>10^{-12}$. In other words, we can omit the effects of $\alpha$ for values lower than about $10^{-12}$, where these results are consistent with those were obtained in Ref. (Bordbar 2006a).

Considering $\alpha=10^{-12}$ and $\Lambda=0$, we plot the neutron star gravitational mass (in solar mass unit $M_{\odot}$ ) as a function of central mass density $\left(\epsilon_{c}\right)$ in Fig. 2 (up diagram). Our results show that at low densities, the calculated neutron star mass exhibits a minimum $\left(\approx 0.12 M_{\odot}\right)$. It can be seen that at high densities, the increasing of gravitational mass becomes very slow, and finally it approaches a limiting value $\left(\approx 1.68 M_{\odot}\right)$. This limiting value is the maximum gravitational mass of neutron star, and its corresponding central density is the highest possible value for the neutron star central density. A star with higher central density would be unstable against the gravitational collapse to a black hole. For mentioned quantities of $\alpha$ and $\Lambda\left(\alpha=10^{-12}\right.$ and $\Lambda=0$ ), we also plot the gravitational mass versus

Table 1 Maximum mass of neutron star and its corresponding radius for various values of $\alpha$ at $\Lambda=0$ and $b=10^{-2}$.

\begin{tabular}{ccc}
\hline \hline$\alpha$ & $M_{\max }\left(M_{\odot}\right)$ & $R(\mathrm{~km})$ \\
\hline \hline $1.33 \times 10^{-11}$ & 0.22 & 12.60 \\
\hline $1.30 \times 10^{-11}$ & 0.81 & 10.01 \\
\hline $1.20 \times 10^{-11}$ & 1.37 & 9.62 \\
\hline $1.10 \times 10^{-11}$ & 1.53 & 9.33 \\
\hline $1.00 \times 10^{-11}$ & 1.60 & 9.11 \\
\hline $5.00 \times 10^{-12}$ & 1.68 & 8.55 \\
\hline $1.00 \times 10^{-12}$ & 1.68 & 8.42 \\
\hline
\end{tabular}


Table 2 Maximum mass of neutron star and its corresponding radius for various values of $\alpha$ and $\Lambda$ at $b=10^{-2}$.

\begin{tabular}{cccc}
\hline \hline$\alpha$ & $M_{\max }\left(M_{\odot}\right)$ & $R(\mathrm{~km})$ & $\Lambda$ \\
\hline \hline $1.00 \times 10^{-12}$ & 0.77 & 6.64 & $1.00 \times 10^{-11}$ \\
\hline $1.00 \times 10^{-13}$ & 0.78 & 6.65 & $1.00 \times 10^{-11}$ \\
\hline $1.00 \times 10^{-14}$ & 0.78 & 6.65 & $1.00 \times 10^{-11}$ \\
\hline $1.00 \times 10^{-12}$ & 1.55 & 8.25 & $1.00 \times 10^{-12}$ \\
\hline $1.00 \times 10^{-13}$ & 1.56 & 8.25 & $1.00 \times 10^{-12}$ \\
\hline $1.00 \times 10^{-11}$ & 1.56 & 9.04 & $1.00 \times 10^{-13}$ \\
\hline $1.00 \times 10^{-13}$ & 1.67 & 8.40 & $1.00 \times 10^{-13}$ \\
\hline $1.00 \times 10^{-14}$ & 1.67 & 8.40 & $1.00 \times 10^{-13}$ \\
\hline $1.00 \times 10^{-11}$ & 1.59 & 9.10 & $1.00 \times 10^{-14}$ \\
\hline $1.00 \times 10^{-12}$ & 1.68 & 8.42 & $1.00 \times 10^{-14}$ \\
\hline $1.00 \times 10^{-13}$ & 1.68 & 8.42 & $1.00 \times 10^{-14}$ \\
\hline & & &
\end{tabular}
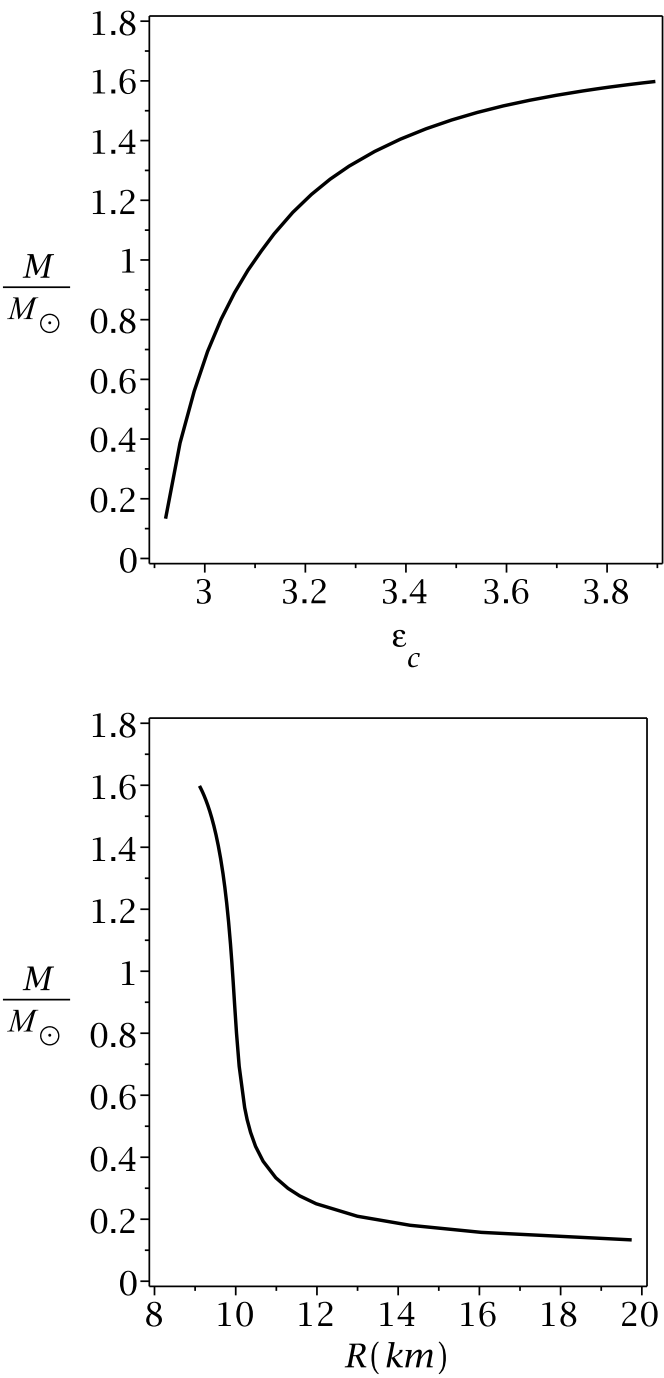

Fig. 2 Gravitational mass versus central mass density, $\epsilon_{c}$ $\left(10^{15} \mathrm{gr} / \mathrm{cm}^{3}\right),(\mathrm{up})$ and radius (down) for $b=10^{-2}, \Lambda=0$ and $\alpha=10^{-12}$.

radius for in Fig. 2 (down diagram). Our results show that for the neutron star, there are a minimum gravitational mass $\left(\approx 0.12 M_{\odot}\right)$ and a maximum gravitational mass $\left(\approx 1.68 M_{\odot}\right)$. It is well known that in this mass region, the equilibrium configuration of neutron stars can exist.

On the other hand, when we consider the effects of ooth $\alpha$ and $\Lambda$ in structure of neutron stars, the results are interesting. Our calculations show that considering a fixed value for $\Lambda$, the maximum mass of the neutron star increases as $\alpha$ decreases. Also, when $\alpha$ is a fixed value, by decreasing $\Lambda$, the maximum mass increases (see Table 2 for more details). Considering the effects of both $\alpha$ and $\Lambda$, simultaneously, we plot the gravitational mass versus radius in Fig. 3. It is notable that for the special values of $\Lambda$ and $\alpha$, our results show an 

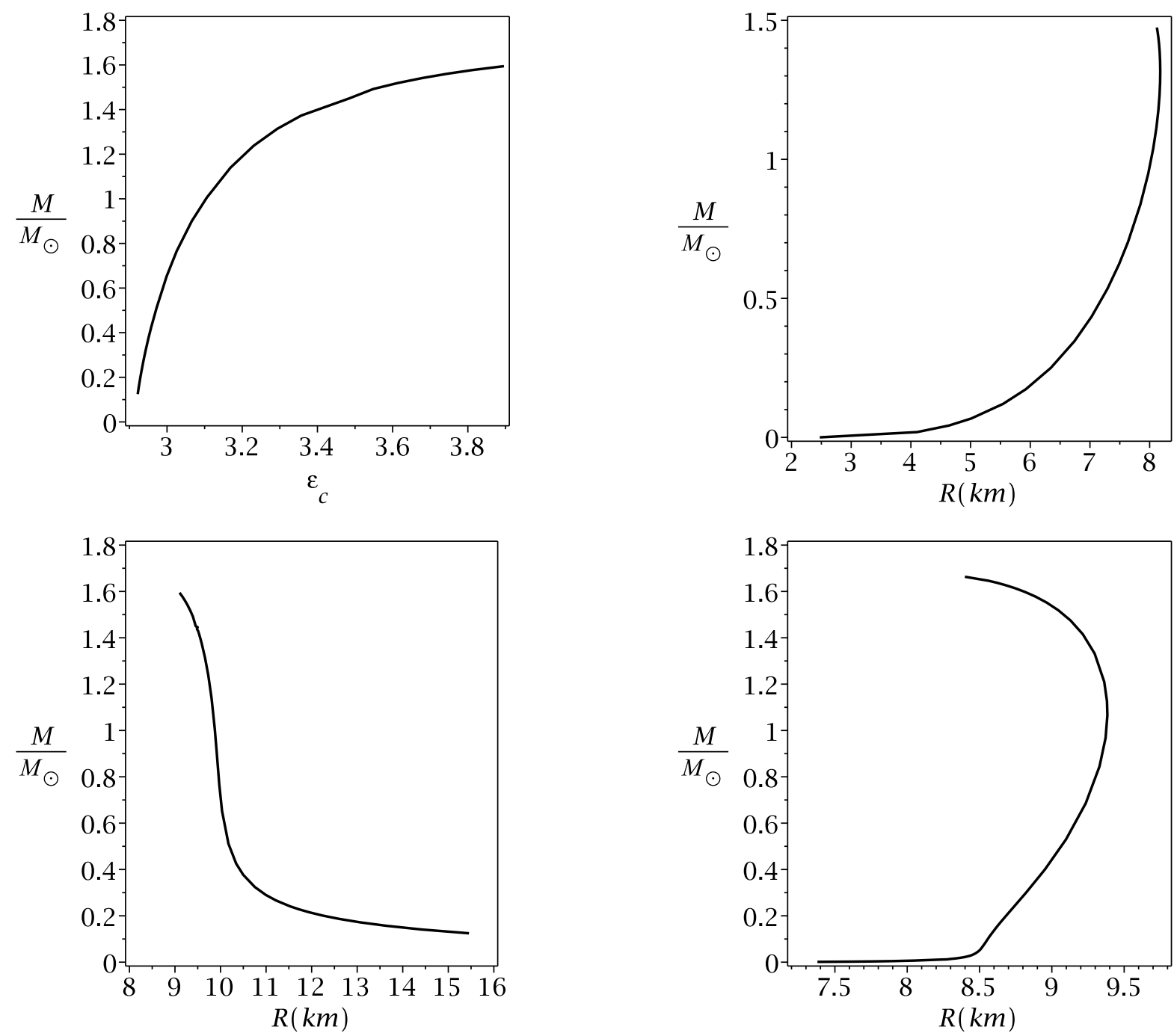

Fig. 3 Gravitational mass versus central mass density, $\epsilon_{c}$ $\left(10^{15} \mathrm{gr} / \mathrm{cm}^{3}\right)$, (up) and radius (down) for $b=10^{-2}, \Lambda=$ $10^{-14}$ and $\alpha=10^{-11}$.

abnormality in the behavior of mass versus radius for neutron star. In other words, for the special values of $\Lambda$ and $\alpha$, we encounter with an interesting behavior. For these cases, with increasing radius, the gravitational mass increases (see Fig. 4), while for a neutron star, with increasing radius, the mass decreases, (see Ref. (Astashenok 2014) for more details). In other words, there are critical values for $\Lambda$ and $\alpha$, so that, the diagram related to gravitational mass versus radius is not similar to the diagram related to ordinary neutron star.

In order to complete our discussion and motivated by AdS spacetimes, we consider negative cosmological constant and collect the results in table 3. We conclude that for a fixed value of dilaton field, increasing the absolute value of negative cosmological constant leads to increasing the maximum mass of the neutron star.

Fig. 4 Gravitational mass versus radius for $b=10^{-2}$ and $\alpha=10^{-12}$ at $\Lambda=1.7 \times 10^{-12}$ (up) and $\Lambda=1.7 \times 10^{-13}$ (down).

In other words, for the negative cosmological constant, one finds that the fraction of maximum mass per solar mass can be larger than 2 .

\section{Closing Remarks}

In this paper, we considered a 4-dimensional spherical symmetric line element and extracted the hydrostatic equilibrium equation of stars in dilaton gravity. We found that for $\alpha=0$ limit, the HEE for dilaton gravity reduces to Einstein- $\Lambda$ one, as one expects. Then, we regarded dilaton field as a correction of EN gravity and showed that $\frac{d P}{d r}$ contains usual TOV equation and an extra dilatonic term. 
Considering the HEE obtained in this paper and using our neutron star matter equation of state, we obtain the structure properties of neutron star. The results showed that as the parameter of dilaton gravity $(\alpha)$ increases, the maximum mass of neutron star decreases. In other words, as $\alpha$ decreases, the effects of dilaton gravity decreases, and the results will be close to the results obtained in Einstein- $\Lambda$ gravity. These results indicated that the dilaton field behaves as an external pressure and prevents the increasing mass of neutron star.

In addition, we regarded negative cosmological constant and found that for a fixed value of dilaton strength, decreasing $\Lambda$ (increasing its absolute value) leads to increasing the maximum mass of the neutron star. In other words, for both positive and negative values of cosmological constant, increasing $\Lambda$ leads to decreasing the gravitational mass. Therefore, one may regard $\Lambda$ as an external pressure which prevents the increasing mass of neutron star. From another point of view, it may be interesting to regard $\Lambda$ as a dynamical pressure (Kubiznak 2012; Hendi 2014b) and relate it to the results of the present work.

Finally, it was proved that dilaton gravity can be transformed to Brans-Dicke theory with a suitable conformal transformation. In addition, it is well-known that a special class of Brans-Dicke theory $\left(w_{B D}=0\right)$ is mathematically equivalent to the $f(R)$ gravity. So, it is interesting to find a relation between obtained results of the present paper with the corresponding $f(R)$ gravity and Brans-Dicke theory. We left these problems for future works.

Acknowledgements We would like to thank the anonymous referees for valuable suggestions. B. E. acknowledges S. Panahiyan and R. Haghbakhsh for helpful discussions. The authors wish to thank Shiraz University Research Council. This work has been supported financially by Research Institute for Astronomy and Astrophysics of Maragha. 


\section{References}

Abbas G. Momeni D., Amir Ali M. Myrzakulov R. and Qaisar S. 2015, Astro. Space Sci. 357, 158

Amarilla L. and Vucetich H. 2010, Int. J. Mod. Phys. A, 25, 3835

Arbanil J. D. V., Lemos J. P. S. and Zanchin V. T. 2013, Phys. Rev. D, 88, 084023

Astashenok A. V., Capozziello S. and Odintsov S. 2013, J. Cosmol. Astropart. Phys., 12, 040

Astashenok A. V., Capozziello S. and Odintsov S. 2014, Phys. Rev. D, 89, 103509

Astashenok A. V., Capozziello S. and Odintsov S. D. 2015, Astrophys. Space Sci, 355, 341

Astashenok A. V., Capozziello S. and Odintsov S. D. 2015, J. Cosmol. Astropart. Phys., 01, 001

Bamba K. and Odintsov S. D. 2008, J. Cosmol. Astropart. Phys., 04, 024

Bordbar G. H. and Modarres M. 1998, Phys. Rev. C 57, 714

Bordbar G. H. and Riazi N. 2002, Astrophys. Space Sci. 282,563

Bordbar G. H. 2004, Int. J. Theor. Phys. 43, 399

Bordbar G. H. and Hayati M. 2006, Int. J. Mod. Phys. A, 21, 1555

Bordbar G. H., Bigdeli M. and Yazdizade T. 2006, Int. J. Mod. Phys. A, 21, 5991

Bordbar G. H. and Rezaei Z. 2013, Res. Astron. Astrophys., 13, 197

Bordbar G. H., Hendi S. H. and Eslam Panah B. 2015, arXiv:1502.02929, gr-qc

Brans C. and Dicke R. H. 1961, Phys. Rev., 124, 925

Brax P. and van de Bruck C. 2003, Class. Quantum Gravit., 20, R201

Cai R. G., Kim S. P. and Wang B. 2007, Phys. Rev. D, 76, 024011

Capozziello S., De Laurentis M., Odintsov S. D. and Stabile A. 2011, Phys. Rev. D, 83, 064004

Capozziello S., De Laurentis M., De Martino I., Formisano M. and Odintsov S. D. 2012, Phys. Rev. D, 85, 044022

Chan K. C. K., Horne J. H. and Mann R. B. 1995, Nucl. Phys. B., 447, 441

Cho Y. M. 1990, Phys. Rev. D, 41, 2462

Cognola G., Elizalde E., Nojiri S., Odintsov S. D., Sebastiani L. and Zerbini S. 2008, Phys. Rev. D, 77, 046009

Corda C. 2009, Europhys. Lett., 86, 20004

Demetrian M. 2006, Gen. Relativ. Gravit., 38, 953

Dehghani M. H. and Farhangkhah N. 2005, Phys. Rev. D, 71, 044008

Dehghani M. H., Sheykhi A. and Hendi S. H. 2008, Phys. Lett. B, 659, 476

Deruelle N. and Farina-Busto L. 1990, Phys. Rev. D, 41, 3696

Diaz A. A. G. 2014, arXiv:1412.5620, gr-qc

Dick R. 1996, arXiv:9609190, hep-th

Doneva D. D., Yazadjiev S. S., Stergioulas N. and Kokkotas K. D. 2013, Phys. Rev. D, 88, 084060

Frieman J. A., Turner M. S. and Huterer D. 2003, Ann. Rev. Astron. Astrophys., 380, 235

Fujii Y. and Maeda K. I. 2003, The Scalar-Tensor Theory of Gravitation, Cambridge University Press

Gergely L. A. 2006, Phys. Rev. D, 74, 024002
Ghodrati M. 2008, J. Cosmol. Astropart. Phys., 04, 024

Giddings S. B. and Strominger A. 1993, Phys. Rev. D, 47, 2454

Goswami R., Nzioki A. M., Maharaj S. D. and Ghosh S. G. 2014, Phys. Rev. D, 90, 084011

Gregory R. and Santos C. 1997, Phys. Rev. D, 56, 1194

Hansraj S., Chilambwe B. and Maharaj S. D. 2015, arXiv:1502.02219, gr-qc

Heap S. R. and Corcoran M. F. 1992, Astrophys. J. 387, 340

Hendi S. H. and Dehghani M. H. 2008, Phys. Lett. B, 666, 116

Hendi S. H., Eslam Panah B. and Mousavi S. M. 2012, Gen. Relativ. Gravit., 44, 835

Hendi S. H., Eslam Panah B. and Saffari R. 2014, Int. J. Mod. Phys. D., 23, 1450088

Hendi S. H., Panahiyan S. and Eslam Panah B. 2014, arXiv:1410.0352, gr-qc

Heydari-Fard M. and Sepangi H. R. 2009, J. Cosmol. Astropart. Phys., 02, 029

Huang Z. G., Lu H. Q. and Fang W. 2007, Int. J. Mod. Phys. D., 16, 1109

Huang Z. G. and Song X. M. 2008, Astrophys. Space Sci., 315,175

Jonker P. G., van der Klis M. and Groot P. J. 2003, Mon. Not. R. Astron. Soc. 339, 663

Jordan P. 1955, Schwerkraft und Weltall, Friedrich Vieweg und Sohn, Brunschweig

Klepac P. and Horsky J. 2002, Gen. Relativ. Gravit., 34, 1979

Kubiznak D., Mann R. B. 2012, JHEP. 07, 033

Lemos J. P. S., Lopes F. J., Quinta G. and Zanchin V. T. 2015, Eur. Phys. J. C., 75, 76

Li X., Wang F. and Cheng K. S. 2012, J. Cosmol. Astropart. Phys., 10, 031

Liang E. P. 1986, Astrophys. J. 304, 682

Ling Y., Niu C., Wu J. P. and Xian Z. Y. 2013, JHEP, 11, 006

Lovelock D. 1971, J. Math. Phys., 12, 498

Lovelock D. 1972, J. Math. Phys., 13, 874

Momeni D., Raza M. and Myrzakulov R. 2014, Eur. Phys. J. Plus 129, 1

Momeni D. and Myrzakov R. 2015, Int. J. Gem. Methods. Mod. Phys. 12, 1550014

Momeni D. Gholizade H. Muhammad R. and Myrzakulov R. 2015, Int. J. Mod. Phys. A 30, 1550093

Narain G., Schaffner-Bielich J. and Mishustin I. N. 2006, Phys. Rev. D, 74, 063003

Nojiri S. and Odintsov S. D. 2011, Phys. Rept., 505, 59

Oliveira A. M., Velten H. E. S., Fabris J. C. and Salako I. G. 2014, Eur. Phys. J. C., 74, 3170

Oppenheimer J. R. and Volkoff G. M. 1939, Phys. Rev., 55, 374

Orellana M., Garcia F., Pannia F. A. T. and Romero G. E. 2013, Gen. Relativ. Gravit., 45, 771

Padmanabhan T. 2003, Phys. Rep., 380, 235

Perlmutter S., et al. 1999, Astrophys. J., 517, 565

Perlmutter S., et al. 1999, Phys. Rev. Lett., 83, 670

Ponce de Leon J. and Cruz N. 2000, Gen. Relativ. Gravit., 32, 1207

Riess A. G., et al. 2004, Astrophys. J., 607, 665 
Shapiro S. and Teukolsky S. 1983, Black Holes, White Dwarfs and Neutron Stars, John Wiley \& Sons, U.S.A

Stoks V. G. J., Klomp R. A. M., Terheggen C. P. F. and de Swart J. J. 1994, Phys. Rev C 49, 2950

Silbar R. R. and Reddy S. 2004, Am. J. Phys., 72, 892

Sotiriou T. P. 2006, Class. Quantum Gravit., 23, 5117

Sotiriou T. P. and Faraoni V. 2010, Rev. Mod. Phys., 82, 451

Tamaki T. and Torii T. 2000, Phys. Rev. D, 62, 061501R

Tolman R. C. 1934, Proc. Nat. Acad. Sc., 20, 169

Tolman R. C. 1939, Phys. Rev., 55, 364

Yamazaki R. and Ida D. 2001, Phys. Rev. D, 64, 024009

Yazadjiev S. S. 2005, Phys. Rev. D, 72, 044006

Yazadjiev S. S., Doneva D. D., Kokkotas K. D. and Staykov K. V. 2014, J. Cosmol. Astropart. Phys., 06, 003

Yazdizadeh T. and Bordbar G. H. 2013, Astrophys., 56, 121

Zhan-Ying Y., De-Cheng Z. and Rui-Hong Y. 2012, Chin. Phys. B, 21, 020401

Quaintrell H. 2003, Astron. Astrophys. 401, 313

Weisberg J. M and Taylor J. H. 1984, Phys. Rev. Lett., 52, 1348

Wiringa R. B., Stoks V. and Schiavilla R. 1995, Phys. Rev. C 51, 38 